CONFORMAL GEOMETRY AND DYNAMICS

An Electronic Journal of the American Mathematical Society

Volume 10, Pages 100-116 (April 6, 2006)

S $1088-4173(06) 00140-8$

\title{
NEVANLINNA THEORETICAL EXCEPTIONAL SETS OF RATIONAL TOWERS AND SEMIGROUPS
}

\author{
YÛSUKE OKUYAMA
}

\begin{abstract}
For a rational tower, i.e., a composition sequence of rational maps, in addition to the algebraic and dynamical exceptional sets, various Nevanlinna theoretical exceptional sets are defined, and as we showed previously in the case of iterations, all of them are the same. In this paper, we extend this result to the cases of a rational tower with summable distortions and a finitely generated rational semigroup. We show that all the exceptional sets of a finitely generated rational semigroup are countable, and all of them are empty if and only if the algebraic one is as well (this being the smallest among them). The countability of exceptional sets is fundamental in the Nevanlinna theory, and their emptiness is important in the complex dynamics.
\end{abstract}

\section{INTRODUCTION}

In recent research on the dynamics of rational semigroups (cf. [25] and [13]), a rational tower naturally appears: From a sequence $h=\left\{h_{k}\right\}_{k \in \mathbb{N}}$ of rational maps, i.e., holomorphic endomorphisms of the Riemann sphere $\hat{\mathbb{C}}$, the rational tower $f=\left\{f_{k}\right\}_{k \in \mathbb{N}}$ is defined as

$$
f_{k}:=h_{k} \circ h_{k-1} \circ \cdots \circ h_{2} \circ h_{1} .
$$

When $h$ is a constant sequence, $f$ is a classical iteration sequence of a rational map. Such an object also appears in the researches on the distribution of zeros of random polynomials (cf. 23 ). Dinh and Sibony deeply studied a rational tower, which they call a random iteration, in a very general context of meromorphic transforms, briefly called MTs (cf. [4] and [5]). In their papers, they studied two problems, one of which is related to the equidistribution and the other to the convergence of the value distributions of MTs. In the equidistribution problem, an exceptional set of values appears, and in the convergence problem, so does a natural summability condition on the MT. In this paper, we shall give a precise description of the exceptional set in the one-dimensional case.

Let $f=\left\{f_{k}\right\}$ be a sequence of rational maps, which is not necessarily a rational tower and is briefly called a rational sequence; let $d_{k}$ be the degree of $f_{k}$.

Received by the editors April 19, 2005 and, in revised form, October 13, 2005.

2000 Mathematics Subject Classification. Primary 30D35; Secondary 37F15, 32H50.

Key words and phrases. Nevanlinna theory, complex dynamics, rational tower, rational semigroup, algebraic exceptional set, Picard exceptional set, dynamical exceptional set, Nevanlinna exceptional set, Valiron exceptional set.

Partially supported by the Ministry of Education, Culture, Sports, Science and Technology of Japan, Grant-in-Aid for Young Scientists (B), 15740085, 2004.

(C)2006 American Mathematical Society Reverts to public domain 28 years from publication 
Definition 1.1 (The Fatou and Julia sets of $f$ ). The Fatou set $F(f)$ is defined as the region of normality of $f$, and the Julia set $J(f)$ its complement in $\hat{\mathbb{C}}$.

The Montel and Picard theorems imply that the behavior of a rational sequence $f$ around $J(f)$ is similar to that of a transcendental meromorphic function on $\mathbb{C}$ around the infinity. As analogues of the Picard exceptional set of a transcendental meromorphic function, we define the two kinds of exceptional sets of a rational sequence.

Notation 1.2 .

(i) The normalized chordal distance for $z, w \in \hat{\mathbb{C}}$ is defined as $[z, w]=$ $|z-w| / \sqrt{\left(1+|z|^{2}\right)\left(1+|w|^{2}\right)}$.

(ii) For $z \in \widehat{\mathbb{C}}$ and $r \in[0,1]$, set $\mathbb{D}_{\sigma}(z, r):=\{w \in \hat{\mathbb{C}} ;[z, w]<r\}$.

(iii) For $p \in \hat{\mathbb{C}}, \delta_{p}$ denotes the Dirac measure at $p$ on $\hat{\mathbb{C}}$.

(iv) For a non-constant rational map $h$, set $h^{*} \delta_{p}:=\sum_{w \in h^{-1} p} \delta_{w}$, where the summation takes into account the local degree of $f$ at each $w \in h^{-1}(p)$ (for a general measure, see Notation 1.17(iii) below).

Definition 1.3 (The Picard and dynamical exceptional sets of $f$ ). The Picard exceptional set is defined as

$$
E_{P}(f):=\hat{\mathbb{C}}-\bigcap_{z \in J(f)} \bigcap_{r \in(0,1]} \bigcup_{k \in \mathbb{N}} f_{k}\left(\mathbb{D}_{\sigma}(z, r)\right),
$$

and the dynamical exceptional set $E(f)$ is defined by the complement in $\hat{\mathbb{C}}$ of the set of all $p \in \hat{\mathbb{C}}$ such that for every weak limit $\mu$ of a subsequence of $\left\{\left(f_{k}\right)^{*} \delta_{p} / d_{k}\right\}$, the relation $\operatorname{supp} \mu \subset J(f)$ holds.

Definition 1.4 (The algebraic exceptional sets of an iteration sequence). For an iteration sequence $\left\{h^{k}\right\}(d:=\operatorname{deg} h>1)$, the algebraic exceptional set is defined as

$$
E_{\text {alg }}\left(\left\{h^{k}\right\}\right):=\{z \in \hat{\mathbb{C}} \text {; periodic with period } \leq 2 \text { and critical with order } d-1\} .
$$

Remark 1.5. It is well known that $E\left(\left\{h^{k}\right\}\right)=E_{P}\left(\left\{h^{k}\right\}\right)=E_{\text {alg }}\left(\left\{h^{k}\right\}\right)$ (cf. [18]). In particular, all of them consist of at most two points, which is regarded as an analogue of the Picard theorem for a transcendental meromorphic function.

From the Nevanlinna theoretical viewpoint, the following exceptional sets are defined. For the Nevanlinna theory, see, for example, [10] and [15].

Notation 1.6. The normalized spherical area measure $\sigma$ is $\mathrm{d} x \mathrm{~d} y /\left(\pi\left(1+|z|^{2}\right)^{2}\right)$ $(z=x+i y)$ on $\hat{\mathbb{C}}$.

Definition 1.7 (The Nevanlinna and Valiron exceptional sets of $f$, cf. [24]). For a point $p \in \hat{\mathbb{C}}$, the Nevanlinna and Valiron defects are defined as

$$
\begin{aligned}
& \delta_{N}(p ; f):=\liminf _{k \rightarrow \infty} \frac{1}{d_{k}} \int \log \frac{1}{\left[f_{k}(w), p\right]} \mathrm{d} \sigma(w) \text { and } \\
& \delta_{V}(p ; f):=\limsup _{k \rightarrow \infty} \frac{1}{d_{k}} \int \log \frac{1}{\left[f_{k}(w), p\right]} \mathrm{d} \sigma(w)
\end{aligned}
$$


respectively. The Nevanlinna and Valiron exceptional sets are defined as

$$
\begin{aligned}
& E_{N}(f):=\left\{p \in \mathbb{C} ; \delta_{N}(p ; f)>0\right\} \quad \text { and } \\
& E_{V}(f):=\left\{p \in \mathbb{C} ; \delta_{V}(p ; f)>0\right\}
\end{aligned}
$$

respectively.

By a standard argument in the Nevanlinna theory, the following holds:

Theorem 1.8 (Casorati-Weierstrass). For a rational sequence $f$ with increasing degrees, $\sigma\left(E_{N}(f)\right)=0$.

Proof.

$$
\begin{aligned}
0 & \leq \int_{\hat{\mathbb{C}}} \liminf _{k \rightarrow \infty}\left(\frac{1}{d_{k}} \int_{\hat{\mathbb{C}}} \log \frac{1}{\left[f_{k}(w), p\right]} \mathrm{d} \sigma(w)\right) \mathrm{d} \sigma(p) \\
& \leq \liminf _{k \rightarrow \infty} \int_{\hat{\mathbb{C}}}\left(\frac{1}{d_{k}} \int_{\hat{\mathbb{C}}}\left(\left(f_{k}\right)^{*} \log \frac{1}{[\cdot, p]}\right)(w) \mathrm{d} \sigma(w)\right) \mathrm{d} \sigma(p) \text { (by the Fatou lemma) } \\
& =\liminf _{k \rightarrow \infty} \frac{1}{d_{k}} \int_{\hat{\mathbb{C}}}\left(\int_{\hat{\mathbb{C}}} \log \frac{1}{[\cdot, p]} \mathrm{d} \sigma(p)\right)\left(\left(f_{k}\right)_{*} \mathrm{~d} \sigma\right)(\cdot) \text { (by the Fubini theorem) } \\
& =\liminf _{k \rightarrow \infty} \frac{1}{d_{k}} \int_{\hat{\mathbb{C}}} \log \frac{1}{[\cdot, p]} \mathrm{d} \sigma(p)=0\left(\text { since } \int_{\hat{\mathbb{C}}} \log \frac{1}{[\cdot, p]} \mathrm{d} \sigma(p) \text { is constant on } \hat{\mathbb{C}}\right) .
\end{aligned}
$$

The following problem is more subtle, and also fundamental from the Nevanlinna theoretical viewpoint.

Problem 1. Are $E_{N}(f)$ and $E_{V}(f)$ countable?

Theorem 1.9 (The countability of exceptional sets [24]). For a rational sequence $f$ with increasing degrees, $E_{V}(f)$ is countable.

We study the analogue of this fundamental problem for finitely generated rational semigroups.

Notation 1.10 (Wordwise dynamics of rational semigroups). Let $G$ be a rational semigroup generated by $m$ rational maps $\left\{h_{j}\right\}_{j=1}^{m}$ of degrees more than one. Each word $x=x_{1} x_{2} \cdots \in \Sigma_{m}:=\{1, \ldots, m\}^{\mathbb{N}}$, which is naturally a compact metric space, is identified with the rational tower $\left\{\langle x\rangle^{k}\right\}_{k \in \mathbb{N}}$, where $\langle x\rangle^{k}:=h_{x_{k}} \circ \cdots \circ h_{x_{1}}$.

Definition 1.11 (Exceptional sets of a rational semigroup). The Picard, Nevanlinna, Valiron, and dynamical exceptional sets of $G$ are defined as $E_{P}(G)$ $:=\bigcup_{x \in \Sigma_{m}} E_{P}(x), E_{N}(G):=\bigcup_{x \in \Sigma_{m}} E_{N}(x), E_{V}(G):=\bigcup_{x \in \Sigma_{m}} E_{V}(x)$ and $E(G):=$ $\bigcup_{x \in \Sigma_{m}} E(x)$ respectively, and the algebraic exceptional one is defined as $E_{\text {alg }}(G):=$ $\bigcup_{g \in G} E_{\text {alg }}\left(\left\{g^{k}\right\}\right)$.

We note that $E_{\mathrm{alg}}(G)$ consists of at most $2 m$ points. On the other hand, since $\Sigma_{m}$ is uncountable, the following is not trivial.

Problem 2. Are $E_{P}(G), E_{N}(G), E_{V}(G)$, and $E(G)$ countable?

We now state our result which answers Problem 2 affirmatively. 
Theorem 1 (Relations between exceptional sets). For a rational semigroup $G$ generated by finitely many rational maps of degrees more than one,

$$
E_{\text {alg }}(G) \subset E_{P}(G) \subset E_{N}(G) \subset E_{V}(G)=E(G) \subset G\left(E_{\text {alg }}(G)\right) .
$$

Here we set $G(S):=\{g(z) ; z \in S, g \in G\}$ for a subset $S$ of $\widehat{\mathbb{C}}$.

Remark 1.12. In the case of iterations, since $E_{\text {alg }}(G)=G\left(E_{\text {alg }}(G)\right)$, Theorem 1 implies that all the exceptional sets are the same (cf. [21] and [22]).

Corollary 1 (The countability and emptiness of the exceptional sets). Under the same assumption as in Theorem 1, all the $E_{\text {alg }}(G), E_{P}(G), E_{N}(G), E_{V}(G), E(G)$ are countable, and all of them are empty if, and only if, one of them is.

Theorem 1 (and Corollary 1) follows from the auxiliary results below.

Notation 1.13 .

(i) $\|\cdot\|$ denotes the Euclidean norm on $\mathbb{C}^{2}$.

(ii) $\pi: \mathbb{C}^{2}-O \rightarrow \hat{\mathbb{C}}$ denotes the canonical projection which maps $\left(z_{0}, z_{1}\right)$ to $z_{1} / z_{0}$ when $z_{0} \neq 0$. Here $O$ is the origin of $\mathbb{C}^{2}$.

Definition 1.14 (Distortion of a rational map). For a rational map $h$ with degree $d$, the distortion of $h$ is defined by

$$
\operatorname{Disto}(h):=\frac{\max \{\|H(Z)\| ;\|Z\|=r\}}{\min \{\|H(Z)\| ;\|Z\|=r\}} \in[1, \infty),
$$

where the radius $r$ is a positive number and $H: \mathbb{C}^{2} \rightarrow \mathbb{C}^{2}$, which is called a lift of $h$, is a homogeneous polynomial map of degree $d$ such that $H(Z)=O$ if and only if $Z=O$, and $\pi \circ H=h \circ \pi$ on $\mathbb{C}^{2}-O$. Clearly $\operatorname{Disto}(h)$ is independent of a radius $r>0$ and a lift of $h$, and hence is well defined.

Definition 1.15 (Summable distortions). Let $f=\left\{f_{k}\right\}$ be a rational tower from a rational sequence $h=\left\{h_{k}\right\}$, and $d_{k}$ the degree of $f_{k}$. Then $f$ is said to be with summable distortions if

$$
S(f):=\sum_{k=1}^{\infty} \frac{\log \left(\operatorname{Disto}\left(h_{k}\right)\right)}{d_{k}}<\infty
$$

Remark 1.16. The sum $S(f)$ seems to be essentially the same as that in [5], Theorem 5.1.

Notation 1.17

(i) For a compact set $K \subset \hat{\mathbb{C}}, B(K)$ denotes the Banach space of all $\mathbb{C}$-valued continuous functions on $K$, and is endowed with the sup-norm on $K$.

(ii) Let $h$ be a non-constant rational map. For every $\phi \in B(\hat{\mathbb{C}})$, its pushforward $h_{*} \phi$ is defined as $\left(h_{*} \phi\right)(z):=\sum_{w \in h^{-1}(z)} \phi(w)(z \in \hat{\mathbb{C}})$, where the summation takes into account the local degree of $h$ at each $w \in h^{-1}(z)$. Then $h_{*}: B(\hat{\mathbb{C}}) \rightarrow B(\hat{\mathbb{C}})$ is bounded and linear.

(iii) The pullback operator $h^{*}: B(\hat{\mathbb{C}})^{\prime} \rightarrow B(\hat{\mathbb{C}})^{\prime}$, where the dual space $B(\hat{\mathbb{C}})^{\prime}$ is identified with the space of all regular measures on $\hat{\mathbb{C}}$, is defined by the transpose of the above $h_{*}$.

(iv) Put $A(h):=h_{*} / \operatorname{deg}(h): B(\hat{\mathbb{C}}) \rightarrow B(\hat{\mathbb{C}})$. 
Theorem 2 (The Brolin theorem for rational towers with summable distortions). Let $f=\left\{f_{k}\right\}$ be a rational tower with summable distortions, and $d_{k}$ the degree of $f_{k}$. Then there exists the unique regular probability measure $\mu_{f}$ on $\hat{\mathbb{C}}$ with $\operatorname{supp} \mu_{f}=$ $J(f)$ such that for every regular probability measure $\mu$ on $\hat{\mathbb{C}}$ with $\mu(E(f))=0$,

$$
\frac{\left(f_{k}\right)^{*} \mu}{d_{k}} \rightarrow \mu_{f}
$$

weakly as $k \rightarrow \infty$.

Remark 1.18. $E(f)$ is the optimal exceptional set in Theorem 2 in that for $\mu=\delta_{p}$, the condition $p \in \hat{\mathbb{C}}-E(f)$ is necessary for (11).

Example 1.19. With no summability condition, the conclusion of Theorem 2 may fail: Consider the rational tower $f$ from the rational sequence $\left\{h_{k}(z)=\lambda_{k} z^{2}\right\}$, where $\lambda_{k}=2^{-2^{k}+2}$ for $k=2 p$ and $\lambda_{k}=2^{2^{k}-1}$ for $k=2 p+1(p \in \mathbb{N})$. Put $P(z)=z^{2}$ and $Q(z)=2 z^{2}$. Then $f_{k}=P^{k}$ for $k=2 p$ and $f_{k}=Q^{k}$ for $k=2 p+1$, thus $J(f)=\{|z|=1\} \cup\{|z|=1 / 2\}$ and $E(f)=\{0, \infty\}$. Then for $a \in \hat{\mathbb{C}}-E(f)$, $\left\{\left(f_{2 p}\right)^{*} \delta_{a} / 2^{2 p}\right\}$ and $\left\{\left(f_{2 p+1}\right)^{*} \delta_{a} / 2^{2 p+1}\right\}$ converges as $p \rightarrow \infty$, and their supports are $\{|z|=1\}$ and $\{|z|=1 / 2\}$ respectively, so (1) does not hold. Indeed, for $k=2 p+1$, we calculate as

$$
\operatorname{Disto}\left(h_{k}\right)=\lambda_{k}\left(1+\frac{1}{\lambda_{k}^{2}+1}\right)^{-1 / 2}>\frac{2^{2^{k}-1}}{2},
$$

which implies that this $f$ is not with summable distortions.

Remark 1.20. In the case of iterations, Theorem 2 was proved by Brolin 2 (for polynomials), Lyubich [16] and Freire-Lopes-Mañé 9. For the other proofs, see also Tortrat [27, Erëmenko-Sodin [6, Hubbard-Papadopol [12, and Fornæss and Sibony [8].

Theorem 2 can be also proven by Fornæss and Sibony's argument in the proof of [8], Theorem 6.1, where they used a crucial contradiction. Alternatively, in this paper, we give a direct proof of Theorem 2, which is, as a merit, conceptually the same as Brolin's original argument.

Theorem 3 (A characterization of the Valiron exceptional set). For a rational tower $f$ with summable distortions,

$$
E_{P}(f) \subset E_{N}(f) \subset E_{V}(f)=E(f) .
$$

Remark 1.21. It is easy to conclude Theorem 2 from Theorem 3 . However, our proof of Theorem 3 is based on Theorem 2, In the case of iterations, we give a proof of Theorem 3, which is independent of Theorem 2 and, hence, gives another proof of it in 22.

The following generalization of the Lyubich ([16]) and Freire-Lopes-Mañé ([9]) theorem is due to Sumi [26] and, in part, to the author.

Theorem 4 (The almost periodicity [26]). Let $G$ be a rational semigroup generated by finitely many rational maps of degrees more than one. Then for every $\phi \in B(\hat{\mathbb{C}})$, $\{A(g) \phi\}_{g \in G}$ is equicontinuous on $\hat{\mathbb{C}}-G\left(E_{\mathrm{alg}}(G)\right)$.

The proof of Theorem 4 has not been published yet, so for the readers' convenience and for completeness, we shall include a proof. 
We remark that since the Valiron and Nevanlinna defects are nice dynamical quantities, it seems to be an interesting problem to generalize the results in this paper in higher dimensions.

\section{Preliminaries from a potential theory}

Notation 2.1. For $Z=\left(z_{0}, z_{1}\right), W=\left(w_{0}, w_{1}\right) \in \mathbb{C}^{2}$, set $Z \wedge W:=z_{0} w_{1}-z_{1} w_{0}$.

Definition 2.2 (Potential and energy). For a positive regular measure $\mu$ on $\mathbb{C}^{2}$ with compact support, its potential is defined by

$$
V^{\mu}:=\int_{\mathbb{C}^{2}} \log |\cdot \wedge W| \mathrm{d} \mu(W): \mathbb{C}^{2} \rightarrow[-\infty,+\infty),
$$

which is a plurisubharmonic function on $\mathbb{C}^{2}$. The energy of $\mu$ is defined by

$$
I^{\mu}:=\int_{\mathbb{C}^{2}} V^{\mu} \mathrm{d} \mu \in[-\infty,+\infty)
$$

Definition 2.3 (Capacity and equilibrium measures). For a compact set $K$ in $\mathbb{C}^{2}$, its capacity is defined by

$$
\operatorname{Cap}(K):=\sup _{\mu ; \mu\left(\mathbb{C}^{2}\right)=1, \operatorname{supp} \mu \subset K} \exp \left(I^{\mu}\right),
$$

which is clearly $\operatorname{SL}(2, \mathbb{C})$-invariant, i.e., $\operatorname{Cap}(K)=\operatorname{Cap}(A(K))$ for $A \in \mathrm{SL}(2, \mathbb{C})$. A regular probability measure $\mu$ with $\operatorname{supp} \mu \subset K$ is called an equilibrium measure on $K$ if it attains the supremum of (2).

Remark 2.4. Since $K$ is compact, by a compactness argument, there always exists an equilibrium measure on $K$.

We introduce domains with such nice properties as in Theorem 2.7below. For the details of the geometric measure theory and the potential theory, see, for example, [7, [19], and [14.

Definition 2.5 (Strictly balanced domains). A domain $D \subset \mathbb{C}^{2}$ is said to be balanced if for every $Z \in D$ and every $\lambda \in \overline{\mathbb{D}}, \lambda Z$ belongs to $D$. A balanced domain $D$ is said to be strictly balanced if for every $\lambda>1, \lambda D \supset \bar{D}$.

Convention (The extended real line). $\overline{\mathbb{R}}:=[-\infty, \infty]$ is endowed with the distance $d_{\mathbb{R}}(x, y)=\left|\tan ^{-1} x-\tan ^{-1} y\right|$, where $\tan ^{-1}( \pm \infty)= \pm \pi / 2$.

Definition 2.6 (The Green function). For a balanced domain $D \subset \mathbb{C}^{2}$, the Green function $G^{D}: \mathbb{C}^{2} \rightarrow[-\infty,+\infty) \subset \overline{\mathbb{R}}$ is defined by

$$
G^{D}(Z):=\log \inf \{\lambda>0 ; Z \in \lambda D\} .
$$

Theorem 2.7 (Azukawa [1]). For a balanced domain $D \subset \mathbb{C}^{2}, G^{D}$ is

(i) continuous if and only if $D$ is strictly balanced, and

(ii) plurisubharmonic on $\mathbb{C}^{2}$ if and only if $D$ is pseudoconvex.

In the rest of this section, let $D$ be a bounded pseudoconvex strictly balanced domain in $\mathbb{C}^{2}$ and fix a compact set $K:=\bar{D}$.

Notation 2.8. Set $\mathrm{d}=\partial+\bar{\partial}$ and $\mathrm{d}^{c}=(i /(2 \pi))(\bar{\partial}-\partial)$, hence $\mathrm{dd}^{c}=(i / \pi) \partial \bar{\partial}$. 
Definition 2.9 (The measure on $\hat{\mathbb{C}}$ derived from $K$ ). Let $\mu_{K}$ be the unique regular probability measure on $\hat{\mathbb{C}}$ such that

$$
\pi^{*} \mu_{K}=\operatorname{dd}^{c} G^{D}
$$

as positive closed $(1,1)$-currents on $\mathbb{C}^{2}$.

Definition 2.10 ( $S^{1}$-invariant measure). For a $\theta \in \mathbb{R}$, put $R_{\theta}(Z)=e^{2 i \pi \theta} Z$ on $\mathbb{C}^{2}$. A regular measure $\mu$ on $\mathbb{C}^{2}$ is said to be $S^{1}$-invariant if $\left\langle\left(R_{\theta}\right)^{*} \phi, \mu\right\rangle=\langle\phi, \mu\rangle$ for every $\theta \in \mathbb{R}$ and every continuous function $\phi$ on $\mathbb{C}^{2}$.

For a regular measure $\mu$ on $\mathbb{C}^{2}$, let $\bar{\mu}$ be the $S^{1}$-invariant regular measure on $\mathbb{C}^{2}$ such that for every continuous function $\phi$ on $\mathbb{C}^{2}$,

$$
\langle\phi, \bar{\mu}\rangle=\left\langle\int_{0}^{1}\left(R_{\rho}\right)^{*} \phi(\cdot) \mathrm{d} \rho, \mu\right\rangle .
$$

The following analogue of the Frostman theorem is useful.

Theorem 2.11 (DeMarco [3, Theorem 3.1).

(i) There exists the unique $S^{1}$-invariant equilibrium measure on $K$.

(ii) A regular probability measure $\mu$ on $\mathbb{C}^{2}$ with $\operatorname{supp} \mu \subset K$ is an equilibrium measure on $K$ if and only if

$$
\operatorname{supp} \mu \subset \partial K \text { and } \pi_{*} \mu=\mu_{K} .
$$

(iii) For every equilibrium measure $\mu$ on $K$,

$$
V^{\mu}=G^{D}+\log \operatorname{Cap}(K)
$$

Definition 2.12 (The $S^{1}$-invariant equilibrium measure). The unique $S^{1}$-invariant equilibrium measure on $K$ is denoted by $\mu^{K}$.

Example 2.13 (The capacity of a ball). Put $\mathbb{B}(r):=\left\{Z \in \mathbb{C}^{2} ;\|Z\| \leq r\right\}$. Then $G^{\mathbb{B}(1)}=\log \|\cdot\|$ and $\mu_{\mathbb{B}(1)}=\sigma$, and it is calculated that $\operatorname{Cap}(\mathbb{B}(1))=e^{-1 / 2}$. Hence the $\mathrm{SL}(2, \mathbb{C})$-invariance implies that $\operatorname{Cap}(\mathbb{B}(r))=r^{2} e^{-1 / 2}$.

\section{A proof of Theorem 2}

In the whole of this section, let $f=\left\{f_{k}\right\}$ be a rational tower from a rational sequence $h=\left\{h_{k}\right\}$ and $d_{k}$ the degree of $f_{k}$.

For each $h_{k}$, choose only such a normalized lift $H_{k}$ as

$$
\max \left\{\left\|H_{k}(Z)\right\| ;\|Z\|=1\right\}=1,
$$

then $H_{k}$ is unique up to the multiplication by a complex number with modulus 1 . $F_{k}:=H_{k} \circ \cdots \circ H_{1}$ is a lift of $f_{k}$, and the sequence $F=\left\{F_{k}\right\}$ is called a normalized lift of $f$.

Since

$$
\left\|H_{k}(\lambda Z)\right\|=|\lambda|^{\operatorname{deg}\left(h_{k}\right)}\left\|H_{k}(Z)\right\|
$$

for every $\lambda \in \mathbb{C},\left\{\left\|F_{k}(Z)\right\|\right\}$ is independent of a choice of a normalized lift of $f$. Hence the non-escaping set

$$
K^{f}:=\left\{Z \in \mathbb{C}^{2} ;\left\|F_{k}(Z)\right\| \not \rightarrow \infty \text { as } k \rightarrow \infty\right\}
$$

is well defined.

Definition 3.1 (The shifts of a rational tower). For each $k \in \mathbb{N} \cup\{0\}, s_{k} f$ denotes the rational tower from the rational sequence $\left\{h_{k+j}\right\}_{j=1}^{\infty}$. 
Assume further that $f$ is with summable distortions.

Lemma 3.2 (The Green function and the non-escaping set).

(i) The limit

$$
G^{f}:=\lim _{k \rightarrow \infty} \frac{\log \left\|F_{k}\right\|}{d_{k}} ; \mathbb{C}^{2} \rightarrow[-\infty,+\infty) \subset \overline{\mathbb{R}}
$$

exists and is uniform on $\mathbb{C}^{2}$. In particular, $G^{f}$ is continuous and plurisubharmonic on $\mathbb{C}^{2}$.

(ii) On $\mathbb{C}^{2}, G^{f}(\lambda Z)=G^{f}(Z)+\log |\lambda|$ for every $\lambda \in \mathbb{C}$, and

$$
-S(f) \leq G^{f}-\log \|\cdot\|=G^{f}(Z /\|Z\|) \leq 0 .
$$

Hence for every $k \in \mathbb{N} \cup\{0\}$,

$$
\mathbb{B}(1) \subset K^{s_{k} f} \subset \mathbb{B}\left(e^{S(f)}\right) .
$$

(iii) $\left\{G^{f}<0\right\}=\left\{Z \in \mathbb{C}^{2}\right.$; $\left.\lim _{k \rightarrow \infty}\left\|F_{k}(Z)\right\| \rightarrow 0\right\}$, which is a bounded pseudoconvex strictly balanced domain, $G^{f}=G^{\left\{G^{f}<0\right\}}, K^{f}=\left\{G^{f} \leq 0\right\}=$ $\overline{\left\{G^{f}<0\right\}}$ and $\mathbb{C}^{2}-K^{f}=\left\{G^{f}>0\right\}=\left\{Z \in \mathbb{C}^{2} ; \lim _{k \rightarrow \infty}\left\|F_{k}(Z)\right\| \rightarrow \infty\right\}$.

(iv) For every $k \in \mathbb{N}$,

$$
\begin{gathered}
d_{k} G^{f}=\left(f_{k}\right)^{*} G^{s_{1} f} \\
\text { on } \mathbb{C}^{2} \text {, and hence } K^{f}=F_{k}^{-1}\left(K^{s_{k} f}\right) \text { and } \partial K^{f}=F_{k}^{-1}\left(\partial K^{s_{k} f}\right) .
\end{gathered}
$$

Proof. By (3) and the normalization, on $\mathbb{C}^{2}$,

$$
-\log \operatorname{Disto}\left(h_{k}\right)+\left(\operatorname{deg} h_{k}\right) \log \|\cdot\| \leq \log \left\|H_{k}\right\| \leq\left(\operatorname{deg} h_{k}\right) \log \|\cdot\|
$$

and by $F_{k}=H_{k} \circ F_{k-1}$ and $d_{k}=\prod_{j=1}^{k} \operatorname{deg} h_{j}$,

$$
-\frac{\log \left(\operatorname{Disto}\left(h_{k}\right)\right)}{d_{k}} \leq \frac{\log \left\|F_{k}\right\|}{d_{k}}-\frac{\log \left\|F_{k-1}\right\|}{d_{k-1}} \leq 0 .
$$

Hence by the summable distortions condition, it follows that $\left\{\left(\log \left\|F_{k}\right\|\right) / d_{k}\right\}$ converges uniformly on $\mathbb{C}^{2}$ as $k \rightarrow \infty$, which proves (ii). The other assertions follow from (4) and (ii).

Definition 3.3 (The measures associated with $f$ ). Put $\mu_{f}:=\mu_{K^{f}}$ and $\mu^{f}:=\mu^{K^{f}}$, where $\mu_{K^{f}}$ and $\mu^{K^{f}}$ are defined as in Definitions 2.9 and 2.12 .

Lemma 3.4 (Invariances of the measures and their supports).

(i) $\mu_{f}$ is non-atomic and $\left(f_{k}\right)^{*} \sigma / d_{k}$ tends to $\mu_{f}$ weakly as $k \rightarrow \infty$.

(ii) For every $k \in \mathbb{N}, d_{k} \mu_{f}=\left(f_{k}\right)^{*} \mu_{s_{k} f}$ and $\left(f_{k}\right)_{*} \mu_{f}=\mu_{s_{k} f}$.

(iii) $\operatorname{supp} \mu_{f}=J(f)$, and hence $\operatorname{supp} \mu^{f}=\partial K^{f} \cap \pi^{-1}(J(f))$.

Proof. Statement (ii) follows from (ii) in Lemma 3.2 and $\pi^{*} \sigma=\operatorname{dd}^{c} \log \|\cdot\|$. Statement (ii) follows from (iv) in Lemma 3.2 .

Concerning (iii), since $\left\{\left(\left(f_{k}\right)^{*} \sigma\right) / \sigma\right\}$ is locally uniformly bounded on $F(f)$ by the Marty theorem, (ii) implies supp $\mu_{f} \subset J(f)$. On the other hand, it is proved by the similar argument to that of [28], Proposition 2.1 that for every point $z \in \hat{\mathbb{C}}-\operatorname{supp} \mu_{f}$, there exists an open neighborhood $V$ of $z$ and a holomorphic section $s: V \rightarrow \mathbb{C}^{2}$ of $\pi$ such that $s(V) \subset \partial K^{f}$. Since $F_{k}(s(V)) \subset F_{k}\left(K^{f}\right) \subset K^{s_{k} f} \subset \mathbb{B}\left(e^{S(f)}\right)$ by (iv) and (iii) in Lemma 3.2 $\left\{F_{k} \circ s\right\}$ is uniformly bounded, hence is a normal family on $V$. Suppose that $\left\{F_{k_{j}} \circ s\right\}$ converges to $\Phi: V \rightarrow \mathbb{C}^{2}$ locally uniformly on $V$. Since $F_{k}(s(V)) \subset F_{k}\left(\partial K^{f}\right) \subset \partial K^{s_{k} f} \subset \mathbb{C}^{2}-\{\|Z\|<1\}$, it holds that $\Phi(V) \in \mathbb{C}^{2}-O$. 
Hence $\pi \circ \Phi$ is well defined and $\left\{f_{k_{j}}=\pi \circ F_{k_{j}} \circ s\right\}$ converges to $\pi \circ \Phi$ locally uniformly on $V$, which proves that $z \in F(f)$. Hence $J(f) \subset \operatorname{supp} \mu_{f}$.

Lemma 3.5 (A weak form of the Brolin theorem). For every $p \in \hat{\mathbb{C}}-E_{N}(f)$, there exists a subsequence of $\left\{\left(f_{k}\right)^{*} \delta_{p} / d_{k}\right\}$ which converges to $\mu_{f}$ weakly.

Proof. For a rational map $h$ and an $p \in \hat{\mathbb{C}}$,

$$
\operatorname{dd}^{c} \log \frac{1}{[h(\cdot), p]}=h^{*}\left(\sigma-\delta_{p}\right),
$$

so for every $C^{\infty}$ function $\phi$ on $\hat{\mathbb{C}}$, there exists a $C_{\phi}>0$ such that for every $p \in \hat{\mathbb{C}}$,

$$
\left\langle\phi, \frac{\left(f_{k}\right)^{*}\left(\sigma-\delta_{p}\right)}{d_{k}}\right\rangle \leq C_{\phi} \frac{1}{d_{k}} \int_{\hat{\mathbb{C}}} \frac{1}{\left[f_{k}(w), p\right]} \mathrm{d} \sigma(w) .
$$

For every $p \in \hat{\mathbb{C}}-E_{N}(f)$, the lim inf of the right-hand side of (6) equals 0 , which with Lemma 3.4(ii) completes the proof.

The following algebraic quantity is first introduced to dynamics in [3].

Definition 3.6 (Resultant). For a homogeneous polynomial map

$$
H(\cdot)=\left(\prod_{i}\left(\cdot \wedge A_{i}\right), \prod_{j}\left(\cdot \wedge B_{j}\right)\right): \mathbb{C}^{2} \rightarrow \mathbb{C}^{2}
$$

of degree $d \geq 2$, its resultant is defined by

$$
\operatorname{Res}(H):=\prod_{i, j} A_{i} \wedge B_{j}
$$

which is well defined and invariant under an $\mathrm{SL}(2, \mathbb{C})$-conjugation of $H$.

Lemma 3.7 (The upper estimate of the potentials). For every $P \in \mathbb{C}^{2}-O$, on $\partial K^{f}$

$$
\limsup _{k \rightarrow \infty} V^{\left(F_{k}\right)^{*} \delta_{P} / d_{k}^{2}} \leq \log \operatorname{Cap}\left(K^{f}\right) .
$$

Proof. Choose $\left\{k_{l}\right\} \subset \mathbb{N}$ as

$$
\lim _{l \rightarrow \infty}\left(-\frac{1}{d_{k_{l}}^{2}} \log \left|\operatorname{Res}\left(F_{k_{l}}\right)\right|\right)=\limsup _{k \rightarrow \infty}\left(-\frac{1}{d_{k}^{2}} \log \left|\operatorname{Res}\left(F_{k}\right)\right|\right) .
$$

From Theorem 1.8 and Lemma 3.5 there exist $\tilde{p}, \tilde{q} \in \mathbb{C}^{2}-O$ with $\pi(\tilde{p}) \neq \pi(\tilde{q})$ and a $\left\{k_{i}:=k_{l_{i}}\right\} \subset\left\{k_{l}\right\}$ such that

$$
\lim _{i \rightarrow \infty} \frac{\left(f_{k_{i}}\right)^{*} \delta_{\pi(\tilde{p})}}{d_{k_{i}}}=\lim _{i \rightarrow \infty} \frac{\left(f_{k_{i}}\right)^{*} \delta_{\pi(\tilde{q})}}{d_{k_{i}}}=\mu_{f}
$$

By an $\operatorname{SL}(2, \mathbb{C})$-conjugation, we assume that $\tilde{p}=(1,0)$ and $\tilde{q}=(0,1)$ without loss of generality.

Furthermore, there exists a $\left\{k_{j}:=k_{i_{j}}\right\} \subset\left\{k_{i}\right\}$ such that $\overline{\left(F_{k_{j}}\right)^{*} \delta_{\tilde{p}} / d_{k_{j}}^{2}}$ and $\overline{\left(F_{k_{j}}\right)^{*} \delta_{\tilde{q}} / d_{k_{j}}^{2}}$ also converge weakly. Then the supports of these limits are contained 
in $\partial K^{f}$ by Lemma 3.2(iii), and for $Z=\tilde{p}, \tilde{q}$,

$$
\begin{aligned}
\pi_{*}\left(\lim _{j \rightarrow \infty} \frac{\overline{\left(F_{k_{j}}\right)^{*} \delta_{Z}}}{d_{k_{j}}^{2}}\right) & =\lim _{j \rightarrow \infty} \pi_{*} \frac{\overline{\left(F_{k_{j}}\right)^{*} \delta_{Z}}}{d_{k_{j}}^{2}} \\
& =\lim _{j \rightarrow \infty} \pi_{*} \frac{\left(F_{k_{j}}\right)^{*} \delta_{Z}}{d_{k_{j}}^{2}}=\lim _{j \rightarrow \infty} \frac{\left(f_{k_{j}}\right)^{*} \delta_{\pi(Z)}}{d_{k_{j}}}=\mu_{f} .
\end{aligned}
$$

Hence by Theorem 2.11(ii) and (iii),

$$
\lim _{j \rightarrow \infty} \frac{\overline{\left(F_{k_{j}}\right)^{*} \delta_{\tilde{p}}}}{d_{k_{j}}^{2}}=\lim _{j \rightarrow \infty} \frac{\overline{\left(F_{k_{j}}\right)^{*} \delta_{\tilde{q}}}}{d_{k_{j}}^{2}}=\mu^{K} .
$$

On the other hand,

$$
\begin{aligned}
\int_{\mathbb{C}^{2}} \int_{\mathbb{C}^{2}} \log |Z \wedge W| \mathrm{d} \overline{\left(\frac{\left(F_{k_{j}}\right)^{*} \delta_{\tilde{p}}}{d_{k_{j}}^{2}}\right)}(Z) \mathrm{d} \overline{\left(\frac{\left(F_{k_{j}}\right)^{*} \delta_{\tilde{q}}}{d_{k_{j}}^{2}}\right)}(W) \\
\quad=\int_{\mathbb{C}^{2}} \int_{\mathbb{C}^{2}} \log |Z \wedge W| \mathrm{d}\left(\frac{\left(F_{k_{j}}\right)^{*} \delta_{\tilde{p}}}{d_{k_{j}}^{2}}\right)(Z) \mathrm{d}\left(\frac{\left(F_{k_{j}}\right)^{*} \delta_{\tilde{q}}}{d_{k_{j}}^{2}}\right) \\
\quad=-\frac{1}{d_{k_{j}}^{2}} \log \left|\operatorname{Res}\left(F_{k_{j}}\right)\right|
\end{aligned}
$$

where the first equality follows by the $S^{1}$-invariance, and the second by a direct calculation.

Consequently, by (8), (99), and an upper-semicontinuity,

$$
\begin{aligned}
\log \operatorname{Cap}\left(K^{f}\right) & =\int_{\mathbb{C}^{2}} \int_{\mathbb{C}^{2}} \log |Z \wedge W| \mathrm{d} \mu^{F}(Z) \mathrm{d} \mu^{F}(W) \\
& \geq \limsup _{k \rightarrow \infty}\left(-\frac{1}{d_{k}^{2}} \log \left|\operatorname{Res}\left(F_{k}\right)\right|\right) .
\end{aligned}
$$

By an $\operatorname{SL}(2, \mathbb{C})$-conjugation, it is enough to show (7) for $P=(1,0) \in \mathbb{C}^{2}$. When $F_{k}(Z)=\left(\prod_{l} Z \wedge A_{l}^{(k)}, \prod_{m} Z \wedge B_{m}^{(k)}\right)$, we directly calculate as

$$
\begin{aligned}
V^{\left(F_{k}\right)^{*} \delta_{P} / d_{k}^{2}} & =\frac{1}{d_{k}} \log \prod_{m}\left|\cdot \wedge B_{m}^{(k)}\right|-\frac{1}{d_{k}^{2}} \log \left|\operatorname{Res}\left(F_{k}\right)\right| \\
& \leq \frac{1}{d_{k}} \log \left\|F_{k}\right\|-\frac{1}{d_{k}^{2}} \log \left|\operatorname{Res}\left(F_{k}\right)\right| .
\end{aligned}
$$

The first term of (111) tends to $G^{f}$, which identically equals 0 on $\partial K^{f}$, as $k \rightarrow \infty$. Hence by (10), (11), and an upper-semicontinuity, on $\partial K^{f}$,

$$
\limsup _{k \rightarrow \infty} V^{\left(F_{k}\right)^{*} \delta_{P} / d_{k}^{2}} \leq \limsup _{k \rightarrow \infty}\left(-\frac{1}{d_{k}^{2}} \log \left|\operatorname{Res}\left(F_{k}\right)\right|\right) \leq \log \operatorname{Cap}\left(K^{f}\right) .
$$

Lemma $3.8\left(\mathbb{C}^{2}\right.$-version of [2], Lemma 15.5). Let $K$ be a compact set in $\mathbb{C}^{2}$ as in Theorem 2.11, let $\left\{\mu_{k}\right\}$ be a sequence of regular probability measures on $\mathbb{C}^{2}$, whose supports are compact, converging to some $\tilde{\mu}$ weakly, and let $\mu$ be an equilibrium measure on $K$. If $\operatorname{supp} \tilde{\mu} \subset \operatorname{supp} \mu$ and $\lim \sup _{k \rightarrow \infty} V^{\mu_{k}} \leq \log \operatorname{Cap}(K)$ on $\operatorname{supp} \mu$, then $\tilde{\mu}$ is also an equilibrium measure on $K$. 
Proof. By the Fatou lemma,

$$
\limsup _{k \rightarrow \infty} \int_{\operatorname{supp} \mu} V^{\mu_{k}} \mathrm{~d} \mu \leq \int_{\operatorname{supp} \mu} \limsup _{k \rightarrow \infty} V^{\mu_{k}} \mathrm{~d} \mu \leq \log \operatorname{Cap}(K) .
$$

On the other hand, by the Fubini theorem, Theorem 2.11(iii) implies that

$$
\limsup _{k \rightarrow \infty} \int_{\operatorname{supp} \mu} V^{\mu_{k}} \mathrm{~d} \mu=\limsup _{k \rightarrow \infty} \int_{\operatorname{supp} \mu} V^{\mu} \mathrm{d} \mu_{k}=\log \operatorname{Cap}(K) .
$$

Therefore $V^{\tilde{\mu}} \geq \lim \sup _{k \rightarrow \infty} V^{\mu_{k}}=\log \operatorname{Cap}(K)$ on $\mu$-almost everywhere $\hat{\mathbb{C}}$, and hence for every $Z_{0} \in \operatorname{supp} \mu(\supset \operatorname{supp} \tilde{\mu}), V^{\tilde{\mu}}\left(Z_{0}\right) \geq \lim \sup _{Z \rightarrow Z_{0}} V^{\tilde{\mu}}(Z) \geq$ $\log \operatorname{Cap}(K)$, which proves $I(\tilde{\mu}) \geq \log \operatorname{Cap}(K)$.

Now we shall complete the proof of Theorem[2. Let $p \in \hat{\mathbb{C}}-E(f)$ and $P \in \pi^{-1}(p)$. Assume that for a $\left\{k_{i}\right\} \subset \mathbb{N},\left\{\left(f_{k_{i}}\right)^{*} \delta_{p} / d_{k_{i}}\right\}$ converges weakly. By the compactness argument, there exists $\left\{k_{j}:=k_{i_{j}}\right\} \subset\left\{k_{i}\right\}$ such that $\tilde{\mu}=\lim _{j \rightarrow \infty}\left(F_{k_{j}}\right)^{*} \delta_{P} / d_{k_{j}}^{2}$ exists. Then supp $\tilde{\mu} \subset \partial K^{f} \cap \pi^{-1}(J(f))=\operatorname{supp} \mu^{F}$ by the definition of $E(f)$ and Lemma 3.4(iii), and hence Lemmas 3.7 and 3.8 imply that $\tilde{\mu}$ is an equilibrium measure on $K^{f}$. Hence by Theorem 2.11(iii),

$$
\lim _{j \rightarrow \infty} \frac{\left(f_{k_{j}}\right)^{*} \delta_{p}}{d_{k_{j}}}=\lim _{j \rightarrow \infty} \pi_{*} \frac{\left(F^{k_{j}}\right)^{*} \delta_{P}}{d_{k_{j}}^{2}}=\pi_{*} \lim _{j \rightarrow \infty} \frac{\left(F^{k_{j}}\right)^{*} \delta_{P}}{d_{k_{j}}^{2}}=\pi_{*} \tilde{\mu}=\mu_{f},
$$

which proves $\lim _{k \rightarrow \infty}\left(f_{k}\right)^{*} \delta_{p} / d_{k}=\mu_{f}$. Theorem 2 follows from an approximation argument and the Fubini theorem for a general $\mu$ with $\mu(E(f))=0$.

\section{A proof of Theorem 3}

Let $f=\left\{f_{k}\right\}$ be a rational tower with summable distortions, and $d_{k}$ the degree of $f_{k}$.

Definition 4.1 (Spherical potential). For a regular measure $\mu$ on $\hat{\mathbb{C}}$, its spherical potential is defined by

$$
V_{\mu}:=\int_{\hat{\mathbb{C}}} \log \frac{1}{[\cdot, w]} \mathrm{d} \mu(w): \hat{\mathbb{C}} \rightarrow[0,+\infty],
$$

which is a $\delta$-subharmonic function on $\hat{\mathbb{C}}$ and $\operatorname{dd}^{c} V_{\mu}=\mu(\hat{\mathbb{C}}) \sigma-\mu$.

Lemma 4.2. The spherical potential $V_{\mu_{f}}$ is continuous (it is first proved by Mañé [17) and $V_{\mu_{f}} \leq 1 / 2+2 S(f)$ on $\hat{\mathbb{C}}$.

Proof. For every $Z \in \mathbb{C}^{2}$,

$$
\begin{aligned}
\left(\pi^{*} V_{\mu_{f}}\right) & (Z) \\
= & \int_{\hat{\mathbb{C}}} \log \frac{1}{[\pi(Z), w]} \mathrm{d} \mu_{f}(w)=\int_{\mathbb{C}^{2}} \log \frac{1}{[\pi(Z), \pi(W)]} \mathrm{d} \mu^{f}(W)\left(\text { by } \pi_{*} \mu^{f}=\mu_{f}\right) \\
= & -V^{\mu^{f}}(Z)+\log \|Z\|+\int_{\mathbb{C}^{2}} \log \|W\| \mathrm{d} \mu^{f}(W)\left(\text { by }[\pi(Z), \pi(W)]=\frac{|Z \wedge W|}{\|Z\|\|W\|}\right) \\
= & -G^{f}(Z /\|Z\|)-\log \operatorname{Cap}\left(K^{f}\right) \\
& +\int_{K^{f}} \log \|W\| \mathrm{d} \mu^{f}(W) \text { (by Theorem 2.11(iii)). }
\end{aligned}
$$


Hence $V_{\mu_{f}}$ is continuous on $\hat{\mathbb{C}}$. By Lemma 3.2(iii), $G^{f}(Z /\|Z\|) \geq-S(f)$ on $\mathbb{C}^{2}$ and $\log \|\cdot\| \leq S(f)$ on $K^{f}$, and also by Example 2.13, $\log \operatorname{Cap}\left(K^{f}\right) \geq \log \operatorname{Cap}(\mathbb{B}(1))=$ $-1 / 2$.

For every $p \in E_{P}(f)$, there exists a $z \in J(f)\left(=\operatorname{supp} \mu_{f}\right.$ by Lemma 3.4) and an $r \in(0,1]$ such that for every weak limit $\mu$ of a subsequence of $\left\{\left(f_{k}\right)^{*} \delta_{p} / d_{k}\right\}$, $\operatorname{supp} \mu \cap \mathbb{D}_{\sigma}(z, r)=\emptyset$. Hence by Lemma 3.5, it follows that $E_{P}(f) \subset E_{N}(f)$.

It is clear that $E_{N}(f) \subset E_{V}(f)$. From ([6), it follows that $E(f) \subset E_{V}(f)$.

We shall show $E_{V}(f) \subset E(f)$. By (5), the following holds on $\hat{\mathbb{C}}$ (cf. [20]):

$$
\log \frac{1}{\left[f_{k}(\cdot), p\right]}=V_{\left(f_{k}\right) *\left(\delta_{p}-\sigma\right)}+\int_{\hat{\mathbb{C}}} \log \frac{1}{\left[f_{k}(w), p\right]} \mathrm{d} \sigma(w) .
$$

Integrating both sides by $\mu_{f}$ over $\hat{\mathbb{C}}$ and dividing them by $d_{k}$, since $\left(f_{k}\right)_{*} \mu_{f}=\mu_{s_{k} f}$ by Lemma 3.4, we have

$$
\frac{V_{\mu_{s_{k} f}}(p)}{d_{k}}=\int_{\hat{\mathbb{C}}} V_{\mu_{f}}(w) \mathrm{d} \frac{\left(f_{k}\right)^{*}\left(\delta_{p}-\sigma\right)}{d_{k}}(w)+\frac{1}{d_{k}} \int_{\hat{\mathbb{C}}} \log \frac{1}{\left[f_{k}(w), p\right]} \mathrm{d} \sigma(w) .
$$

For every $k \in \mathbb{N}$, by Lemma 4.2, $V_{\mu_{s_{k} f}} \leq 1 / 2+2 S\left(s_{k} f\right) \leq 1 / 2+2 S(f)$ on $\hat{\mathbb{C}}$ and $V_{\mu_{f}}$ is continuous on $\hat{\mathbb{C}}$. Hence for every $p \in \hat{\mathbb{C}}-E(f)$, both the left-hand side and the first term of the right-hand side (by Theorem 2) tend to 0 as $k \rightarrow \infty$, so $p \in \hat{\mathbb{C}}-E_{V}(f)$.

\section{A proof of Theorem 4}

Let $G$ be a rational semigroup considered in Notation 1.10, and fix an $\epsilon>0$.

Notation 5.1. Put $C:=\max _{1 \leq j \leq m} \#\left\{\right.$ critical points of $\left.h_{j}\right\}$ and for every $l \in \mathbb{N}$,

$$
Z_{l}:=\bigcup_{x \in \Sigma_{m}} \bigcup_{k=1}^{l}\left\{\text { critical values of }\langle x\rangle^{k}\right\} \text {. }
$$

For $x \in \Sigma_{m}$ and $k \in \mathbb{N}$, we denote $\langle x\rangle_{k}:=h_{x_{1}} \circ \cdots \circ h_{x_{k}}$. Hence $\operatorname{deg}\langle x\rangle_{k}=$ $\operatorname{deg}\langle x\rangle^{k}$.

Choose an $l=l(\epsilon) \in \mathbb{N}$ so large that $C / 2^{l-1}<\epsilon / 2$. For $x \in \Sigma_{m}$ and a simply connected domain $U \subset \hat{\mathbb{C}}-Z_{l}$, let $\sigma_{k}=\sigma_{k}(x, U)(k \in \mathbb{N})$ be the number of all single-valued branches of $\left(\langle x\rangle_{k}\right)^{-1}$ on $U$. Then $\sigma_{l}=\operatorname{deg}\langle x\rangle^{l}$ and for every $r \in \mathbb{N}$, $\sigma_{l+r} \geq \operatorname{deg}\left(h_{l+r}\right)\left(\sigma_{l+r-1}-C\right)$, hence

$$
\begin{gathered}
\sigma_{l+r} \geq \operatorname{deg}\langle x\rangle^{l+r}-C \sum_{k=1}^{r} \prod_{i=0}^{k-1} \operatorname{deg}\left(h_{l+r-i}\right), \quad \text { so } \\
\frac{\operatorname{deg}\langle x\rangle^{l+r}-\sigma_{l+r}}{\operatorname{deg}\langle x\rangle^{l+r}} \leq \frac{C}{\operatorname{deg}\langle x\rangle^{l}} \sum_{k=1}^{r}\left(\frac{1}{2}\right)^{r-k} \leq \frac{2 C}{2^{l}}<\frac{\epsilon}{2} .
\end{gathered}
$$

Notation 5.2. For $x \in \Sigma_{m}$ and $k \in \mathbb{N}$, put $\langle x\rangle^{-k}:=\left(\langle x\rangle^{k}\right)^{-1}, s_{k} x:=x_{k+1} x_{k+2} \cdots \in$ $\Sigma_{m}$, and $d:=\max _{1 \leq j \leq m} \operatorname{deg}\left(h_{j}\right)$.

Since $G$ is generated by $m$ elements, for every $x \in \Sigma_{m}$ and every $y \in \hat{\mathbb{C}}-$ $G\left(E_{\text {alg }}(G)\right), \#\langle x\rangle^{-(2 m+1)}(y) \geq 2$ without multiplicity. Hence there exists a $\tau=$ $\tau(\epsilon) \in \mathbb{N}$ such that for every $x \in \Sigma_{m}$ and every $y \in \hat{\mathbb{C}}-G\left(E_{\text {alg }}(G)\right), \#\langle x\rangle^{-\tau}(y) \geq$ $\# Z_{l(\epsilon)}$ without multiplicity. 
For every $y \in \hat{\mathbb{C}}-G\left(E_{\text {alg }}(G)\right)$ and every $x \in \Sigma_{m}$, defined are inductively $n(j, x ; y)$, $S_{j, g}^{x}(y)$ and $S_{j, b}^{x}(y)(j \in \mathbb{N} \cup\{0\})$ as follows: Put $n(0, x ; y):=0, S_{0, g}^{x}(y):=\emptyset$ and $S_{0, b}^{x}(y):=\{y\}$. For each $j \in \mathbb{N} \cup\{0\}$, put

$$
\begin{gathered}
n(j+1, x ; y):=n(j, x ; y)+N(j, x ; y), \text { where } \\
N(j, x ; y):=\min \left\{n \in \mathbb{N} ; \operatorname{deg}\left\langle s_{n(j, x ; y)} x\right\rangle^{n} \geq d^{\tau}\right\}(\geq \tau),
\end{gathered}
$$

for every $z \in S_{j, b}^{x}(y)$, choose a point $p_{z} \in\left(\left\langle s_{n(j, x ; y)} x\right\rangle_{N(j, x ; y)}\right)^{-1}(z) \subset \hat{\mathbb{C}}-Z_{l}$, and put $S_{j+1, g}^{x}(y)=\left\{p_{z} ; z \in S_{j, b}^{x}(y)\right\}$ and $S_{j+1, b}^{x}(y):=\left(\left\langle s_{n(j, x ; y)} x\right\rangle_{N(j, x ; y)}\right)^{-1}\left(S_{j, b}^{x}(y)\right)-$ $S_{j+1, g}^{x}(y)$. Then $\# S_{0, g}^{x}(y)=0, \# S_{0, b}^{x}(y)=1$, and for every $j \in \mathbb{N}$,

$$
\begin{gathered}
\# S_{j+1, g}^{x}(y)=\# S_{j, b}^{x}(y) \quad \text { and } \\
\# S_{j, b}^{x}(y) \leq\left(\operatorname{deg}\left\langle s_{n(j-1, x ; y)} x\right\rangle^{N(j-1, x ; y)}-1\right) \# S_{j-1, b}^{x}(y) .
\end{gathered}
$$

Choose a $k=k(\epsilon) \in \mathbb{N}$ so large that $\left(1-1 / d^{\tau(\epsilon)+1}\right)^{k(\epsilon)}<\epsilon$. Then for every $y \in \hat{\mathbb{C}}-G\left(E_{\text {alg }}(G)\right)$, by (14),

$$
\begin{gathered}
\frac{\# S_{j, b}^{x}(y)}{\operatorname{deg}\langle x\rangle^{n(j, x ; y)}} \leq \prod_{i=0}^{j-1}\left(1-\frac{1}{\operatorname{deg}\left\langle s_{n(i, x ; y)} x\right\rangle^{N(i, x ; y)}}\right) \leq\left(1-\frac{1}{d^{\tau+1}}\right)^{j}, \text { so } \\
\frac{\# S_{k, b}^{x}(y)}{\operatorname{deg}\langle x\rangle^{n(k, x ; y)}}<\epsilon .
\end{gathered}
$$

Fix a $\phi \in B(\hat{\mathbb{C}})$. For every $z \in \hat{\mathbb{C}}-Z_{l}$ and every $\delta>0$ where $\mathbb{D}_{\sigma}(z, \delta) \subset \hat{\mathbb{C}}-Z_{l}$, it follows from (12) that for every such $g \in G$ as a composition of more than $l(\epsilon)$ elements of $\left\{h_{j}\right\}_{j=1}^{m}$ and every $z^{\prime} \in \mathbb{D}_{\sigma}(z, \delta)$,

$$
\left|(A(g) \phi)(z)-(A(g) \phi)\left(z^{\prime}\right)\right| \leq \sup _{h}\left|\phi(h(z))-\phi\left(h\left(z^{\prime}\right)\right)\right|+2 \cdot \frac{\epsilon}{2} \sup _{\widehat{\mathbb{C}}}|\phi|,
$$

where in the first term of the right-hand side, $h$ is a single-valued branch of $\left(g^{-1}\right) \mid \mathbb{D}_{\sigma}(z, \delta)$, and the supremum is taken over all such branches.

Fix an $x \in \Sigma_{m}$ and a $y \in \hat{\mathbb{C}}-G\left(E_{\text {alg }}(G)\right)$. The following is useful for estimating the first term of the right-hand side of (17):

Theorem 5.3 (Hinkkanen and Martin [11, Theorem 2.1). For every domain $U \subset$ $\hat{\mathbb{C}}$, the family $\bigcup_{g \in G}$ \{single valued branches of $\left.\left(g^{-1}\right) \mid U\right\}$ is normal on $U$.

By (17) and Theorem 5.3, if $\delta=\delta(\epsilon, x, y, \phi)>0$ is small enough, then for every $g \in G$ and every $z \in \bigcup_{j=1}^{k} S_{j, g}^{x}(y)$, which is finite, it holds that $\mathbb{D}_{\sigma}(z, \delta) \subset \hat{\mathbb{C}}-Z_{l}$ and

$$
\sup _{z^{\prime} \in \mathbb{D}_{\sigma}(z, \delta)}\left|(A(g) \phi)(z)-(A(g) \phi)\left(z^{\prime}\right)\right|<2 \epsilon \sup _{\hat{\mathbb{C}}}|\phi| .
$$

Choose a sufficiently small $\delta_{1}=\delta_{1}(\epsilon, x, y)>0$ such that for every $y^{\prime} \in \mathbb{D}_{\sigma}\left(y, \delta_{1}\right)$ and every $j \in\{1, \ldots, k\}$, there exist such indices $\left\{y_{i}(j)\right\}_{i=1}^{\operatorname{deg}\langle x\rangle^{n(j, x ; y)}}$ of $\left(\langle x\rangle_{n(j, x ; y)}\right)^{-1}(y)$ and $\left\{y_{i}^{\prime}(j)\right\}_{i=1}^{\operatorname{deg}\langle x\rangle^{n(j, x ; y)}}$ of $\left(\langle x\rangle_{n(j, x ; y)}\right)^{-1}\left(y^{\prime}\right)$ that for every $i \in\left\{1, \ldots, \operatorname{deg}\langle x\rangle^{n(j, x ; y)}\right\}$,

$$
y_{i}^{\prime}(j) \in \mathbb{D}_{\sigma}\left(y_{i}(j), \delta\right) .
$$


Then for every $y^{\prime} \in \mathbb{D}_{\sigma}\left(y, \delta_{1}\right)-G\left(E_{\text {alg }}(G)\right)$ and every $g \in G$,

$$
\begin{aligned}
& \left|\left(A\left(\langle x\rangle_{n(k, x ; y)} \circ g\right) \phi\right)(y)-\left(A\left(\langle x\rangle_{n(k, x ; y)} \circ g\right) \phi\right)\left(y^{\prime}\right)\right| \\
& =\left|\sum_{i=1}^{\operatorname{deg}\langle x\rangle^{n(k, x ; y)}}(A(g) \phi)\left(y_{i}(k)\right)-(A(g) \phi)\left(y_{i}^{\prime}(k)\right)\right| / \operatorname{deg}\langle x\rangle^{n(k, x ; y)} \\
& \leq \sum_{j=1}^{k}\left|\sum_{\left(\left\langle s_{n(j, x ; y)} x\right\rangle_{n(k, x ; y)-n(j, x ; y)}\right)^{-1}\left(S_{j, g}^{x}(y)\right)} \frac{(A(g) \phi)\left(y_{i}(k)\right)-(A(g) \phi)\left(y_{i}^{\prime}(k)\right)}{\operatorname{deg}\langle x\rangle^{n(k, x ; y)}}\right| \\
& \quad+\sum_{i: y_{i}(k) \in S_{k, b}^{x}(y)} \frac{\left|(A(g) \phi)\left(y_{i}(k)\right)-(A(g) \phi)\left(y_{i}^{\prime}(k)\right)\right|}{\operatorname{deg}\langle x\rangle^{n(k, x ; y)}} \\
& \leq(d+1) \cdot 2 \epsilon \sup _{\hat{\mathbb{C}}}|\phi|
\end{aligned}
$$

since by (19), (18), (13), and (15),

$$
\begin{aligned}
\text { (20) }) \leq & \sum_{j=1}^{k} \sum_{i: y_{i}(j) \in S_{j, g}^{x}(y)} \frac{\left|\left(A(\overbrace{\left\langle s_{n(j, x ; y)} x\right\rangle_{n(k, x ; y)-n(j, x ; y)} \circ g}) \phi\right)\left(y_{i}(j)\right)-(A(*) \phi)\left(y_{i}^{\prime}(j)\right)\right|}{\operatorname{deg}\langle x\rangle^{n(j, x ; y)}} \mid \\
& \leq \sum_{j=1}^{k} \frac{\# S_{j, g}^{x}(y)}{\operatorname{deg}\langle x\rangle^{n(j, x ; y)}} \cdot 2 \epsilon \sup _{\hat{\mathbb{C}}}|\phi|=\sum_{j=1}^{k} \frac{\# S_{j-1, b}^{x}(y)}{\operatorname{deg}\langle x\rangle^{n(j, x ; y)}} \cdot 2 \epsilon \sup _{\hat{\mathbb{C}}}|\phi| \\
& \leq \sum_{j=1}^{k} \frac{1}{d^{\tau}}\left(1-\frac{1}{d^{\tau+1}}\right)^{j-1} \cdot 2 \epsilon \sup _{\hat{\mathbb{C}}}|\phi| \leq d \cdot 2 \epsilon \sup _{\hat{\mathbb{C}}}|\phi|,
\end{aligned}
$$

and by (16),

$$
\text { (21) } \leq \frac{\# S_{k, b}^{x}(y)}{\operatorname{deg}\langle x\rangle^{n(k, x ; y)}} \cdot 2 \sup _{\hat{\mathbb{C}}} \mid\left(A(g) \phi\left|\leq 2 \epsilon \sup _{\hat{\mathbb{C}}}\right| \phi \mid .\right.
$$

Now we shall complete the proof of Theorem 4, Since $\Sigma_{m}$ is compact and for every $x \in \Sigma_{m},\left\{x^{\prime} \in \Sigma_{m} ; x_{j}^{\prime}=x_{j}\right.$ for $\left.1 \leq j \leq n(k(\epsilon), x ; y)\right\}$ is open, there exist $x(1 ; y), \ldots, x(M ; y) \in \Sigma_{m}$ such that

$$
\Sigma_{m}=\bigcup_{i=1}^{M}\left\{x \in \Sigma_{m} ; x_{j}=(x(i ; y))_{j} \text { for } 1 \leq j \leq n(k(\epsilon), x(i ; y) ; y)\right\} .
$$

For every $g \in\left\{\langle x\rangle^{k} ; x \in \Sigma_{m}, k>\max _{1 \leq i \leq M} n(k(\epsilon), x(i) ; y)\right\}$, there exist $i \in$ $\{1, \ldots, M\}$ and $g_{0} \in G$ such that $g=\langle x(i)\rangle_{n(k(\epsilon), x(i ; y) ; y)} \circ g_{0}$, and hence for every $y^{\prime} \in \mathbb{D}_{\sigma}\left(y, \delta_{1}\right)-G\left(E_{\text {alg }}(G)\right)$,

$$
\left|(A(g) \phi)(y)-(A(g) \phi)\left(y^{\prime}\right)\right| \leq(d+1) \cdot 2 \epsilon \sup _{\hat{\mathbb{C}}}|\phi| .
$$

Since $G-\left\{\langle x\rangle^{k} ; x \in \Sigma_{m}, k>\max _{1 \leq i \leq M} n(k(\epsilon), x(i) ; y)\right\}$ is finite, if $\delta_{1}$ is small enough, (22) holds for every $g \in G$, and every $y^{\prime} \in \mathbb{D}_{\sigma}\left(y, \delta_{1}\right)-G\left(E_{\text {alg }}(G)\right)$. 


\section{A proof of Theorem 1}

We continue to use the notation in Section 5 . Since $G$ is finitely generated, every rational tower $\left\{\langle x\rangle^{k}\right\}\left(x \in \Sigma_{m}\right)$ is with summable distortions, so Theorem 3 is applicable to it. Hence only the proof of $E(G) \subset G\left(E_{\mathrm{alg}}(G)\right)$ is left.

For the proof of the following, see [12], pp. 335-336.

Lemma 6.1. For every $x \in \Sigma_{m}$ and every smooth probability measure $\nu$,

$$
\frac{\left(\langle x\rangle^{k}\right)^{*} \nu}{\operatorname{deg}\langle x\rangle^{k}} \rightarrow \mu_{x}
$$

weakly as $k \rightarrow \infty$.

Fix a $p \in \hat{\mathbb{C}}-G\left(E_{\text {alg }}(G)\right)$. Let $\eta_{n} \rightarrow 0$ as $n \rightarrow \infty$. Then for every $\phi \in B(\hat{\mathbb{C}})$, by Theorem 4 there exists such an $\left\{\epsilon_{n}\right\} \subset \mathbb{R}_{\geq 0}$ that for every $n \in \mathbb{N}$ and every $g \in G$,

$$
\sup _{w \in \mathbb{D}_{\sigma}\left(p, \epsilon_{n}\right)}|(A(g) \phi)(w)-(A(g) \phi)(p)|<\eta_{n} .
$$

Choose smooth probability measures $\left\{\nu_{n}\right\}$ on $\hat{\mathbb{C}}$ such that $\nu_{n} \rightarrow \delta_{p}$ weakly as $n \rightarrow \infty$ and $\operatorname{supp} \nu_{n} \subset \mathbb{D}_{\sigma}\left(p, \epsilon_{n}\right)$. Then by (24), for every $x \in \Sigma_{m}$,

$$
\begin{aligned}
& \left|\left\langle\phi, \frac{\left(\langle x\rangle^{k}\right)^{*} \nu_{n}}{\operatorname{deg}\langle x\rangle^{k}}-\frac{\left(\langle x\rangle^{k}\right)^{*} \delta_{p}}{\operatorname{deg}\langle x\rangle^{k}}\right\rangle\right| \\
& \quad \leq \int_{\mathbb{D}_{\sigma}\left(p, \epsilon_{n}\right)}\left|\left(A\left(\langle x\rangle^{k}\right) \phi\right)(w)-\left(A\left(\langle x\rangle^{k}\right) \phi\right)(p)\right| \mathrm{d} \nu_{n}(w)<\eta_{n} .
\end{aligned}
$$

From this and Lemma 6.1, for every $n \in \mathbb{N}$ and every limit $L$ of a subsequence of $\left\{\left\langle\phi,\left(\langle x\rangle^{k}\right)^{*} \delta_{p} / \operatorname{deg}\langle x\rangle^{k}\right\rangle\right\}$, it follows that $\left|\left\langle\phi, \mu_{f}\right\rangle-L\right| \leq \eta_{n}$, so that $L=\left\langle\phi, \mu_{f}\right\rangle$. Hence (23) holds for $\nu=\delta_{p}$, which concludes $p \in \hat{\mathbb{C}}-E(G)$.

Remark 6.2. In [26, Sumi presented Theorem 4 under the assumption that there exists a compact set $K \subset \widehat{\mathbb{C}}-E_{\text {alg }}(G)$ such that $\bigcup_{g \in G} g^{-1}(K) \subset K$. In particular, $K \subset \hat{\mathbb{C}}-G\left(E_{\text {alg }}(G)\right)$. Then, as a bonus, for every $\phi \in B(K),\{A(g) \phi\}_{g \in G}$ is not only equicontinuous but also uniformly bounded by $\sup _{K}|\phi|$ on $K$. Hence by the Ascoli-Arzelà theorem, it further follows that (23) holds uniformly for every regular probability measure $\nu$ with $\operatorname{supp} \nu \subset K$.

\section{ACKNOWLEDGMENT}

The author would like to express his gratitude to Professor Tien-Cuong Dinh for kindly introducing to the author his and Sibony's recent research on meromorphic transforms, to Professor Hiroki Sumi for invaluable discussions on rational semigroups, and to Professors Masahiko Taniguchi and Toshiyuki Sugawa for useful comments.

\section{REFERENCES}

[1] K. Azukawa, Hyperbolicity of circular domains, Tohoku Math. J. (2) 35, 3 (1983), 403-413. MR0711356 (85c:32041)

[2] H. Brolin, Invariant sets under iteration of rational functions, Ark. Mat. 6 (1965), 103-144. MR0194595 (33:2805) 
[3] L. DeMarco, Dynamics of rational maps: Lyapunov exponents, bifurcations, and capacity, Math. Ann. 326, 1 (2003), 43-73. MR1981611 (2004f:32044)

[4] T.-C. Dinh, Suites d'applications méromorphes multivaluées et courants laminaires, J. Geom. Anal. 15, 2 (2005), 207-227. MR2152480

[5] T.-C. Dinh and N. Sibony, Value distribution of meromorphic transforms and applications, math.DS/0306095.

[6] A. E. Erëmenko and M. L. Sodin, Iterations of rational functions and the distribution of the values of Poincaré functions, Teor. Funktsiu Funktsional. Anal. i Prilozhen. 53 (1990), 18-25. MR 1077218 (92d:30016)

[7] H. Federer, Geometric measure theory, Die Grundlehren der mathematischen Wissenschaften, Band 153, Springer-Verlag New York Inc., New York (1969). MR0257325 (41:1976)

[8] J. E. Fornaess and N. Sibony, Complex dynamics in higher dimension. II, Modern methods in complex analysis (Princeton, NJ, 1992), Vol. 137 of Ann. of Math. Stud., Princeton Univ. Press, Princeton, NJ (1995), 135-182. MR1369137 (97g:32033)

[9] A. Freire, A. Lopes, and R. Mañé, An invariant measure for rational maps, Bol. Soc. Brasil. Mat. 14, 1 (1983), 45-62. MR0736568 (85m:58110b)

[10] A. Hinkkanen, Sharp error term in the Nevanlinna theory, Complex differential and functional equations (Mekrijärvi, 2000), Vol. 5 of Univ. Joensuu Dept. Math. Rep. Ser., Univ. Joensuu, Joensuu (2003), 51-79. MR1968110 (2004i:30021)

[11] A. Hinkkanen and G. J. Martin, Some properties of semigroups of rational functions, XVIth Rolf Nevanlinna Colloquium (Joensuu, 1995), de Gruyter, Berlin (1996), 53-58. MR1427070 (97i:30036)

[12] J. H. Hubbard and P. Papadopol, Superattractive fixed points in $\mathbf{C}^{n}$, Indiana Univ. Math. J. 43, 1 (1994), 321-365. MR.1275463 (95e:32025)

[13] M. Jonsson, Ergodic properties of fibered rational maps, Ark. Mat. 38, 2 (2000), 281-317. MR1785403 (2002k:37073)

[14] M. Klimek, Pluripotential theory, Vol. 6 of London Mathematical Society Monographs. New Series, The Clarendon Press Oxford University Press, New York (1991), Oxford Science Publications. MR.1150978 (93h:32021)

[15] I. Laine, Nevanlinna theory and complex differential equations, Vol. 15 of de Gruyter Studies in Mathematics, Walter de Gruyter \& Co., Berlin (1993). MR1207139 (94d:34008)

[16] M. J. Ljubich, Entropy properties of rational endomorphisms of the Riemann sphere, Ergodic Theory Dynam. Systems 3, 3 (1983), 351-385. MR0741393(85k:58049)

[17] R. Mañé, The Hausdorff dimension of invariant probabilities of rational maps, Dynamical systems, Valparaiso 1986, Vol. 1331 of Lecture Notes in Math., Springer, Berlin (1988), 86117. MR0961095 (90j:58073)

[18] S. Morosawa, Y. Nishimura, M. Taniguchi, and T. Ueda, Holomorphic dynamics, Vol. 66 of Cambridge Studies in Advanced Mathematics, Cambridge University Press, Cambridge (2000), Translated from the 1995 Japanese original and revised by the authors. MR 1747010 (2002c:37064)

[19] J. Noguchi and T. Ochiai, Geometric function theory in several complex variables, Vol. 80 of Translations of Mathematical Monographs, American Mathematical Society, Providence, RI (1990), Translated from the Japanese by Noguchi. MR1084378 (92e:32001)

[20] Y. Okuyama, Nevanlinna, Siegel, and Cremer, Indiana Univ. Math. J. 53, 3 (2004), 755-763. MR2086699

[21] Y. Okuyama, Complex dynamics, value distributions, and potential theory, Ann. Acad. Sci. Fenn. Math. 30, 2 (2005), 303-311. MR 2173366

[22] Y. Okuyama, Valiron, Nevanlinna and Picard exceptional sets of iterations of rational functions, Proc. Japan Acad. Ser. A Math. Sci. 812 (2005), 23-26. MR2126072 (2006a:30026)

[23] B. Shiffman and S. Zelditch, Distribution of zeros of random and quantum chaotic sections of positive line bundles, Comm. Math. Phys. 200, 3 (1999), 661-683. MR1675133(2001j:32018)

[24] M. Sodin, Value distribution of sequences of rational functions, Entire and subharmonic functions, Vol. 11 of Adv. Soviet Math., Amer. Math. Soc., Providence, RI (1992), 7-20. MR1188001 (93k:30050)

[25] H. Sumi, Skew product maps related to finitely generated rational semigroups, Nonlinearity 13, 4 (2000), 995-1019. MR.1767945 (2001g:37060)

[26] H. Sumi, unpublished manuscript (2001). 
[27] P. Tortrat, Aspects potentialistes de l'itération des polynômes, Séminaire de Théorie du Potentiel, Paris, No. 8, Vol. 1235 of Lecture Notes in Math., Springer, Berlin (1987), 195-209.

[28] T. Ueda, Fatou sets in complex dynamics on projective spaces, J. Math. Soc. Japan 46, 3 (1994), 545-555. MR1276837 (95d:32030)

Department of Mathematics, Faculty of Science, Kanazawa University, Kanazawa 920-1192 JAPAN

Current address: Department of Mathematics and Statistics, University of Helsinki, P.O. Box 68 (Gustaf Hällströmin katu 2b), FI-00014 Helsinki, Finland

E-mail address: yusuke.okuyama@helsinki.fi 